\title{
Gause Symmetry and Howe Duality in 4D Conformal Field Theory Models
}

\author{
I. Todorov
}

To Jiri Niederle on the occasion of his 70th birthday

\begin{abstract}
It is known that there are no local scalar Lie fields in more than two dimensions. Bilocal fields, however, which naturally arise in conformal operator product expansions, do generate infinite Lie algebras. It is demonstrated that these Lie algebras of local observables admit (highly reducible) unitary positive energy representations in a Fock space. The multiplicity of their irreducible components is governed by a compact gauge group. The mutually commuting observable algebra and gauge group form a dual pair in the sense of Howe. In a theory of local scalar fields of conformal dimension two in four space-time dimensions the associated dual pairs are constructed and classified.
\end{abstract}

The paper reviews joint work of B. Bakalov, N. M. Nikolov, K.-H. Rehren and the author.

\section{Introduction}

We review results of $[32,33,34,35,36]$ and $[2,3]$ on $4 \mathrm{D}$ conformal field theory (CFT) models, which can be summed up as follows. The requirement of global conformal invariance (GCI) in compactified Minkowski space together with the Wightman axioms [41] implies the Huygens principle (Eq. (3.6) below) and rationality of correlation functions [32]. A class of 4D GCI quantum field theory models gives rise to a (reducible) Fock space representation of a pair consisting of an infinite dimensional Lie algebra $\mathcal{L}$ and a commuting with it compact Lie group $U$. The state space $\mathcal{F}$ splits into a direct sum of irreducible $\mathcal{L} \times U$ modules, so that each irreducible representation (IR) of $\mathcal{L}$ appears with a multiplicity equal to the dimension of an associated IR of $U$. The pair $(\mathcal{L}, U)$ illustrates a interconnects two independent developments: (i) it appears as a reductive dual pair, [16, 17], within (a central extension of) an infinite dimensional symplectic Lie algebra; (ii) it provides a representation theoretic realization of the Doplicher-Haag-Roberts' (DHR) theory of superselection sectors and compact gauge groups, [8, 14]. I shall first briefly recall Howe's and DHR's theories; then (in Sect. 2) I will explain how some 2D CFT techniques can be extended to four space-time dimensions (in spite of persistent doubts that this is at all possible). After these preliminaries we shall proceed with our survey of 4D CFT models and associated infinite dimensional Lie algebras which relate the two independent developments.

\subsection{Reductive dual pairs}

The notion of a (reductive) dual pair was introduced by Roger Howe in an influential preprint of the 1970's that was eventually published in [17]. It was previewed in two earlier papers of Howe, [15, 16], highlightening the role of the Heisenberg group and the applications of dual pairs to physics. For Howe a dual pair, the counterpart for groups and for Lie algebras of the mutual commutants of von Neumann algebras ([14]), is a (highly structured) concept that plays a unifying role in such widely different topics as Weil's metaplectic group approach [44] to $\theta$ functions and automorphic forms (an important chapter in number theory) and the quantum mechanical Heisenberg group along with the description of massless particles in terms of the ladder representations of $U(2,2)$ [31], among others (in physics).

Howe begins in [16] with a $2 n$-dimensional real symplectic manifold $\mathcal{W}=\mathcal{V}+\mathcal{V}^{\prime}$ where $\mathcal{V}$ is spanned by $\mathrm{n}$ symbols $a_{i}, i=1, \ldots, n$, called annihilation operators and $\mathcal{V}^{\prime}$ is spanned by their conjugate, the creation operators $a_{i}^{*}$ satisfying the canonical commutation relations (CCR)

$$
\left[a_{i}, a_{j}\right]=0=\left[a_{i}^{*}, a_{j}^{*}\right],\left[a_{i}, a_{j}^{*}\right]=\delta_{i j} .
$$

The commutator of two elements of the real vector space $\mathcal{W}$ being a real number it defines a (nondegenerate, skew-symmetric) bilinear form on it which vanishes on $\mathcal{V}$ and on $\mathcal{V}^{\prime}$ separately and for which $\mathcal{V}^{\prime}$ appears as the dual space to $\mathcal{V}$ (the space of linear functionals on $\mathcal{V}$ ). The real symplectic Lie algebra $s p(2 n, \mathbb{R})$ spanned by antihermitean quadratic combinations of $a_{i}$ and $a_{j}^{*}$ acts by commutators on $\mathcal{W}$ preserving its reality and the above bilinear form. This action extends to the Fock space $\mathcal{F}$ (unitary, irreducible) representation of the CCR. It is, however, only exponentiated to the double cover of $S p(2 n, \mathbb{R})$, the metaplectic group $M p(2 n)$ (which is not a matrix group i.e., has no faithful finite-dimensional representation; we can view its Fock space, called by Howe [16] oscillator representation as the defining one). Two subgroups $G$ and $G^{\prime}$ of $M p(2 n)$ are said to form a (reductive) dual pair if they act reductively on $\mathcal{F}$ (that is automatic for 
a unitary representation like the one considered here) and each of them is the full centralizer of the other in $M p(2 n)$. The oscillator representation of $M p(2 n)$ displays a minimality property, [19] that keeps attracting the attention of both physicists and mathematicians see e.g. $[25,12,26]$.

\subsection{Local observables determine a compact gauge group}

Observables (unlike charge carrying fields) are left invariant by (global) gauge transformations. This is, in fact, part of the definition of a gauge symmetry or a superselection rule as explained by Wick, Wightman and Wigner [45]. It required the non-trivial vision of Rudolf Haag to predict in the 1960's that a local net of obsevable algebras should determine the compact gauge group that governs the structure of its superselection sectors (for a review and references to the original work - see [14]). It took over 20 years and the courage and dedication of Haag's (then) young collaborators, Doplicher and Roberts [8] to carry out this program to completion. They proved that all superselection sectors of a local QFT $\mathcal{A}$ with a mass gap are contained in the vacuum representation of a canonically associated (graded local) field extension $\mathcal{E}$, and they are in a one-to-one correspondence with the unitary irreducible representations (IR) of a compact gauge group $G$ of internal symmetries of $\mathcal{E}$, so that $\mathcal{A}$ consists of the fixed points of $\mathcal{E}$ under $G$. The pair $(\mathcal{A}, \mathcal{G})$ in $\mathcal{E}$ provides a general realization of a dual pair in a local quantu theory.

\section{How do 2D CFT methods work in higher dimensions?}

A number of reasons are given why 2-dimensional conformal field theory is, in a way, exceptional so that extending its methods to higher dimensions appears to be hopeless.

1. The $2 D$ conformal group is infinite dimensional: it is the direct product of the diffeomorphism groups of the left and right (compactified) light rays. (In the euclidean picture it is the group of analytic and antianalytic conformal mappings.) By contrast, for $D>2$, according to the Liouville theorem, the quantum mechanical conformal group in $D$ space-time dimensions is finite (in fact, $\left.\frac{(D+1)(D+2)}{2}\right)$-dimensional: it is (a covering of) the spin group $\operatorname{Spin}(D, 2)$.

2. The representation theory of affine Kac-Moody algebras [20] and of the Virasoro algebra [23] plays a crucial role in constructing soluble $2 D$ models of (rational) CFT. There are, on the other hand, no local Lie fields in higher dimensions: after an inconclusive attempt by Robinson [39] (criticized in [28]) this was proven for scalar fields by Baumann [4].

3. The light cone in two dimensions is the direct product of two light rays. This geometric fact is the basis of splitting $2 D$ variables into rightand left-movers' chiral variables. No such splitting seems to be available in higher dimensions.

4. There are chiral algebras in $2 D \mathrm{CFT}$ whose local currents satisfy the axioms of vertex algebras ${ }^{1}$ and have rational correlation functions. It was believed for a long time that they have no physically interesting higher dimensional CFT analogue.

5. Furthermore, the chiral currents in a $2 D \mathrm{CFT}$ on a torus have elliptic correlation functions [46], the 1-point function of the stress energy tensor appearing as a modular form (these can be also interpreted as finite temperature correlation functions and a thermal energy mean value on the Riemann sphere). Again, there seemed to be no good reason to expect higher dimensional analogues of these attractive properties.

We shall argue that each of the listed features of $2 D$ CFT does have, when properly understood, a higher dimensional counterpart.

1. The presence of a conformal anomaly (a non-zero Virasoro central charge $c$ ) tells us that the infinite conformal symmetry in $1+1$ dimension is, in fact, broken. What is actually used in $2 D$ CFT are the (conformal) operator product expansions (OPEs) which can be derived for any $\mathrm{D}$ and allow to extend the notion of a primary field (for instance with respect to the stress-energy tensor).

2. For $D=4$, infinite dimensional Lie algebras are generated by bifields $V_{i j}\left(x_{1}, x_{2}\right)$ which naturally arise in the OPE of a (finite) set of (say, hermitean, scalar) local fields $\phi_{i}$ of dimension $d(>1)$ :

$$
\begin{aligned}
& \left(x_{12}^{2}\right)^{d} \phi_{i}\left(x_{1}\right) \phi_{j}\left(x_{2}\right)= \\
& N_{i j}+x_{12}^{2} V_{i j}\left(x_{1}, x_{2}\right)+O\left(\left(x_{12}^{2}\right)^{2}\right), \\
& x_{12}=x_{1}-x_{2}, \quad x^{2}=\mathbf{x}^{2}-x^{0^{2}}, \\
& N_{i j}=N_{j i} \in \mathbb{R}
\end{aligned}
$$

where $V_{i j}$ are defined as (infinite) sums of OPE contributions of (twist two) conserved local tensor currents (and the real symmetric matrix $\left(N_{i j}\right)$ is positive definite). We say more on this in what follows (reviewing results of $[33,34,35,36,2,3]$ ).

3. We shall exhibit a factorization of higher dimensional intervals by using the following parametrization of the conformally compactified space-time $([43,42,37,38])$ :

\footnotetext{
${ }^{1}$ As a mathematical subject vertex algebras were anticipated by I. Frenkel and V. Kac [11] and introduced by R. Borcherds [5]; for reviews and further references see e.g. [21] [10]
} 


$$
\begin{aligned}
& \bar{M}=\left\{z_{\alpha}=e^{i t} u_{\alpha}, \alpha=1, \ldots, D ;\right. \\
& \left.t, u_{\alpha} \in \mathbb{R} ; u^{2}=\sum_{\alpha=1}^{D} u_{\alpha}^{2}=1\right\}=\frac{\mathbb{S}^{D-1} \times \mathbb{S}^{1}}{\{1,-1\}} .
\end{aligned}
$$

The real interval between two points $z_{1}=e^{i t_{1}} u_{1}$, $z_{2}=e^{i t_{2}} u_{2}$ is given by:

$$
\begin{aligned}
z_{12}^{2}\left(z_{1}^{2} z_{2}^{2}\right)^{-1 / 2}= & 2\left(\cos t_{12}-\cos \alpha\right)= \\
& -4 \sin t_{+} \sin t_{-}, \quad z_{12}=z_{1}-z_{2} \\
t_{ \pm}= & 1 / 2\left(t_{12} \pm \alpha\right), \\
u_{1} \cdot u_{2}= & \cos \alpha, \quad t_{12}=t_{1}-t_{2} .
\end{aligned}
$$

Thus $t_{+}$and $t_{-}$are the compact picture counterparts of "left" and "right" chiral variables (see [38]). The factorization of $2 D$ cross ratios into chiral parts again has a higher dimensional analogue [7]:

$$
\begin{aligned}
s & :=\frac{x_{12}^{2} x_{34}^{2}}{x_{13}^{2} x_{24}^{2}}=u_{+} u_{-} \\
t & :=\frac{x_{14}^{2} x_{23}^{2}}{x_{13}^{2} x_{24}^{2}}=\left(1-u_{+}\right)\left(1-u_{-}\right), x_{i j}=x_{i}-x_{j}
\end{aligned}
$$

which yields a separation of variables in the d'Alembert equation (cf. Remark 2.1) One should, in fact, be able to derive the factorization (2.6) from (2.4).

4. It turns out that the requirement of global conformal invariance (GCI) in Minkowski space together with the standard Wightman axioms of local commutativity and energy positivity entails the rationality of correlation functions in any even number of space-time dimensions [32]. Indeed, GCI and local commutativity of Bose fields (for space-like separations of the arguments) imply the Huygens principle and, in fact, the strong (algebraic) locality condition

$$
\begin{aligned}
& \left(x_{12}^{2}\right)^{n}\left[\phi_{i}\left(x_{1}\right), \phi_{j}\left(x_{2}\right)\right]=0 \\
& \text { for } n \text { sufficiently large, }
\end{aligned}
$$

a condition only consistent with the theory of free fields for an even number of space time dimensions. It is this Huygens locality condition which allows the introduction of higher dimensional vertex algebras [37, 38, 1].

5. Local GCI fields have elliptic thermal correlation functions with respect to the (differences of) conformal time variables in any even number of space-time dimensions; the corresponding energy mean values in a Gibbs (KMS) state (see e.g. [14]) are expressed as linear combinations of modular forms [38].

The rest of the paper is organized as follows. In Sect. 3 we reproduce the general form of the 4-point function of the bifield $V$ and the leading term in its conformal partial wave expansion. The case of a theory of scalar fields of dimension $d=2$ is singled out, in which the bifields (and the unit operator) close a commutator algebra. In Sect. 4 we classify the arising infinite dimensional Lie algebras $\mathcal{L}$ in terms of the three real division rings $\mathbb{F}=\mathbb{R}, \mathbb{C}, \mathbb{H}$. In Sect. 5 we formulate the main result of [2] and [3] on the Fock space representations of the Lie algebra $\mathcal{L}(\mathbb{F})$ coupled to the (dual, in the sense of Howe [16]) compact gauge group $U(N, \mathbb{F})$ where $N$ is the central charge of $\mathcal{L}$.

\section{Four-point functions and conformal partial wave expansions}

The conformal bifields $V\left(x_{1}, x_{2}\right)$ of dimension $(1,1)$ which arise in the OPE (2.2) (as sums of integrals of conserved tensor currents) satisfy the d'Alembert equation in each argument [34]; we shall call them harmonic bifields. Their correlation functions depend on the dimension $d$ of the local scalar fields $\phi$. For $d=1$ one is actually dealing with the theory of a free massless field. We shall, therefore, assume $d>1$. A basis $\left\{f_{\nu i}, \nu=0,1, \ldots, d-2, i=1,2\right\}$ of invariant amplitudes $F(s, t)$ such that

$$
\begin{gathered}
\left\langle 0\left|V_{1}\left(x_{1}, x_{2}\right) V_{2}\left(x_{3}, x_{4}\right)\right| 0\right\rangle=\frac{1}{\rho_{13} \rho_{24}} F(s, t), \\
\rho_{i j}=x_{i j}^{2}+i 0 x_{i j}^{0}, \quad x^{2}=x^{2}-\left(x^{0}\right)^{2}
\end{gathered}
$$

is given by

$$
\begin{aligned}
\left(u_{+}-u_{-}\right) f_{\nu 1}(s, t) & =\frac{u_{+}^{\nu+1}}{\left(1-u_{+}\right)^{\nu+1}}-\frac{u_{-}^{\nu+1}}{\left(1-u_{-}\right)^{\nu+1}}, \\
\left(u_{+}-u_{-}\right) f_{\nu 2}(s, t) & =(-1)^{\nu}\left(u_{+}^{\nu+1}-u_{-}^{\nu+1}\right), \\
\nu & =0,1, \ldots, d-2,
\end{aligned}
$$

where $u_{ \pm}$are the "chiral variables" (2.6);

$$
\begin{aligned}
f_{01} & =\frac{1}{t}, \quad f_{02}=1 ; \\
f_{11} & =\frac{1-s-t}{t^{2}}, f_{12}=t-s-1 ; \\
f_{21} & =\frac{(1-t)^{2}-s(2-t)+s^{2}}{t^{3}}, \\
f_{\nu 2}(s, t) & =\frac{1}{t} f_{\nu 1}\left(\frac{s}{t}, \frac{1}{t}\right)
\end{aligned}
$$

$f_{\nu, i}, i=1,2$ corresponding to single pole terms [36] in the 4-point correlation functions $w_{\nu i}\left(x_{1}, \ldots, x_{4}\right)=$ $f_{\nu i}(s, t) / \rho_{13} \rho_{24}$ :

$$
\begin{aligned}
& w_{01}=\frac{1}{\rho_{14} \rho_{23}}, \\
& w_{02}=\frac{1}{\rho_{13} \rho_{24}} ;
\end{aligned}
$$




$$
\begin{aligned}
w_{11}= & \frac{\rho_{13} \rho_{24}-\rho_{14} \rho_{23}-\rho_{12} \rho_{34}}{\rho_{14}^{2} \rho_{23}^{2}}, \\
w_{12}= & \frac{\rho_{14} \rho_{23}-\rho_{13} \rho_{24}-\rho_{12} \rho_{34}}{\rho_{13}^{2} \rho_{24}^{2}} ; \\
w_{21}= & \frac{\left(\rho_{13} \rho_{24}-\rho_{14} \rho_{23}\right)^{2}}{\rho_{14}^{3} \rho_{23}^{3}}- \\
& \frac{\rho_{12} \rho_{34}\left(2 \rho_{13} \rho_{24}-\rho_{14} \rho_{23}\right)+\rho_{12}^{2} \rho_{34}^{2}}{\rho_{14}^{3} \rho_{23}^{3}}, \\
w_{22}= & \frac{\left(\rho_{14} \rho_{23}-\rho_{13} \rho_{24}\right)^{2}}{\rho_{13}^{3} \rho_{24}^{3}}- \\
& \frac{\rho_{12} \rho_{34}\left(2 \rho_{14} \rho_{23}-\rho_{13} \rho_{24}\right)+\rho_{12}^{2} \rho_{34}^{2}}{\rho_{13}^{3} \rho_{24}^{3}} .
\end{aligned}
$$

We have $w_{\nu 2}=P_{34} w_{\nu 1}\left(=P_{12} w_{\nu 1}\right)$ where $P_{i j}$ stands for the substitution of the arguments $x_{i}$ and $x_{j}$. Clearly, for $x_{1}=x_{2}$ (or $s=0, t=1$ ) only the amplitudes $f_{0 i}$ contribute to the 4-point function (3.1). It has been demonstrated in [35] that the lowest angular momentum $(\ell)$ contribution to $f_{\nu i}$ corresponds to $\ell=\nu$. The corresponding OPE of the bifield $V$ starts with a local scalar field $\phi$ of dimension $d=2$ for $\nu=0$; with a conserved current $j_{\mu}$ (of $d=3$ ) for $\nu=1$; with the stress energy tensor $T_{\lambda \mu}$ for $\nu=2$. Indeed, the amplitude $f_{\nu 1}$ admits an expansion in twist two $^{2}$ conformal partial waves $\beta_{\ell}(s, t)[6]$ starting with (for a derivation see [35], Appendix B)

$$
\begin{aligned}
\beta_{\nu}(s, t) & =\frac{G_{\nu+1}\left(u_{+}\right)-G_{\nu+1}\left(u_{-}\right)}{u_{+}-u_{-}}, \\
G_{\mu}(u) & =u^{\mu} F(\mu, \mu ; 2 \mu ; u) .
\end{aligned}
$$

Remark 3.1 Eqs. (3.2) (3.5) provide examples of solutions of the d'Alambert equation in any of the arguments $x_{i}, i=1,2,3,4$. In fact, the general conformal covariant (of dimension 1 in each argument) such solution has the form of the right hand side of (3.1) with

$$
F(s, t)=\frac{f\left(u_{+}\right)-f\left(u_{-}\right)}{u_{+}-u_{-}} .
$$

Remark 3.2 We note that albeit each individual conformal partial wave is a transcendental function (like (3.5)) the sum of all such twist two contributions is the rational function $f_{\nu 1}(s, t)$.

It can be deduced from the analysis of 4-point functions that the commutator algebra of a set of harmonic bifields generated by OPE of scalar fields of dimension $d$ can only close on the $V$ 's and the unit operator for $d=2$. In this case the bifields $V$ are proven, in addition, to be Huygens bilocal [36].

Remark 3.3 In general, irreducible positive energy representations of the (connected) conformal group are labeled by triples $\left(d ; j_{1}, j_{2}\right)$ including the dimension $d$ and the Lorentz weight $\left(j_{1}, j_{2}\right)\left(2 j_{i} \in \mathbb{N}\right),[29]$. It turns out that for $d=3$ there is a spin-tensor bifield of weight $((3 / 2 ; 1 / 2,0),(3 / 2 ; 0,1 / 2))$ whose commutator algebra does close; for $d=4$ there is a conformal tensor bifield of weight $((2 ; 1,0),(2 ; 0,1))$ with this property. These bifields may be termed lefthanded: they are analogues of chiral $2 D$ currents; a set of bifields invariant under space reflections would also involve their righthanded counterparts (of weights $((3 / 2 ; 0,1 / 2),(3 / 2 ; 1 / 2,0))$ and $((2 ; 0,1),(2 ; 1,0))$, respectively).

\section{Infinite dimensional Lie algebras and real division rings}

Our starting point is the following result of [36].

Proposition 4.1. The harmonic bilocal fields $V$ arising in the OPEs of a (finite) set of local hermitean scalar fields of dimension $d=2$ can be labeled by the elements $M$ of an unital algebra $\mathcal{M} \subset \operatorname{Mat}(L, \mathbb{R})$ of real matrices closed under transposition, $M \rightarrow{ }^{t} M$, in such a way that the following commutation relations (CR) hold:

$$
\begin{aligned}
& {\left[V_{M_{1}}\left(x_{1}, x_{2}\right), V_{M_{2}}\left(x_{3}, x_{4}\right)\right]=} \\
& \Delta_{13} V_{{ }^{t} M_{1} M_{2}}\left(x_{2}, x_{4}\right)+\Delta_{24} V_{M_{1}{ }^{t} M_{2}}\left(x_{1}, x_{3}\right)+ \\
& \Delta_{23} V_{M_{1} M_{2}}\left(x_{1}, x_{4}\right)+\Delta_{14} V_{M_{2} M_{1}}\left(x_{3}, x_{2}\right)+ \\
& \operatorname{tr}\left(M_{1} M_{2}\right) \Delta_{12,34}+\operatorname{tr}\left({ }^{t} M_{1} M_{2}\right) \Delta_{12,43} ;
\end{aligned}
$$

here $\Delta_{i j}$ is the free field commutator, $\Delta_{i j}:=\Delta_{i j}^{+}-$ $\Delta_{j i}^{+}$, and $\Delta_{12, i j}=\Delta_{1 i}^{+} \Delta_{2 j}^{+}-\Delta_{i 1}^{+} \Delta_{j 2}^{+}$where $\Delta_{i j}^{+}=$ $\Delta^{+}\left(x_{i}-x_{j}\right)$ is the 2-point Wightman function of a free massless scalar field.

We call the set of bilocal fields closed under the CR (4.1) a Lie system. The types of Lie systems are determined by the corresponding t-algebras - i.e., real associative matrix algebras $\mathcal{M}$ closed under transposition. We first observe that each such $\mathcal{M}$ can be equipped with a Frobenius inner product

$$
<M_{1}, M_{2}>=\operatorname{tr}\left({ }^{t} M_{1} M_{2}\right)=\sum_{i j}\left(M_{1}\right)_{i j}\left(M_{2}\right)_{i j},
$$

which is symmetric, positive definite, and has the property $<M_{1} M_{2}, M_{3}>=<M_{1}, M_{3}{ }^{t} M_{2}>$. This implies that for every right ideal $\mathcal{I} \subset \mathcal{M}$ its orthogonal complement is again a right ideal while its transposed ${ }^{t} \mathcal{I}$ is a left ideal. Therefore, $\mathcal{M}$ is a semisimple algebra so that every module over $\mathcal{M}$ is a direct sum of irreducible modules.

Let now $\mathcal{M}$ be irreducible. It then follows from the Schur's lemma (whose real version [27] is richer

\footnotetext{
${ }^{2}$ The twist of a symmetric traceless tensor is defined as the difference between its dimension and its rank. All conserved symmetric tensors in $4 D$ have twist two.
} 
but less popular than the complex one) that its commutant $\mathcal{M}^{\prime}$ in $\operatorname{Mat}(L, \mathbb{R})$ coincides with one of the three real division rings (or not necessarily commutative fields): the fields of real and complex numbers $\mathbb{R}$ and $\mathbb{C}$, and the noncommutative division ring $\mathbb{H}$ of quaternions. In each case the Lie algebra of bilocal fields is a central extension of an infinite dimensional Lie algebra that admits a discrete series of highest weight representations ${ }^{3}$.

It was proven, first in the theory of a single scalar field $\phi$ (of dimension two) [33], and eventually for an arbitrary set of such fields [36], that the bilocal fields $V_{M}$ can be written as linear combinations of normal products of free massless scalar fields $\varphi_{i}(x)$ :

$$
V_{M}\left(x_{1}, x_{2}\right)=\sum_{i, j=1}^{L} M^{i j}: \varphi_{i}\left(x_{1}\right) \varphi_{j}\left(x_{2}\right): .
$$

For each of the above types of Lie systems $V_{M}$ has a canonical form, namely

$$
\begin{aligned}
& \mathbb{R}: V\left(x_{1}, x_{2}\right)=\sum_{i=1}^{N}: \varphi_{i}\left(x_{1}\right) \varphi_{i}\left(x_{2}\right):, \\
& \mathbb{C}: W\left(x_{1}, x_{2}\right)=\sum_{j=1}^{N}: \varphi_{j}^{*}\left(x_{1}\right) \varphi_{j}\left(x_{2}\right):, \\
& \mathbb{H}: Y\left(x_{1}, x_{2}\right)=\sum_{m=1}^{N}: \varphi_{m}^{+}\left(x_{1}\right) \varphi_{m}\left(x_{2}\right):
\end{aligned}
$$

where $\varphi_{i}$ are real, $\varphi_{j}$ are complex, and $\varphi_{m}$ are quaternionic valued fields (corresponding to (3.2) with $L=$ $N, 2 N$, and $4 N$, respectively). We shall denote the associated infinite dimensional Lie algebra by $\mathcal{L}(\mathbb{F}), \mathbb{F}=$ $\mathbb{R}, \mathbb{C}$, or $\mathbb{H}$.

Remark 4.1 We note that the quaternions (represented by $4 \times 4$ real matrices) appear both in the definition of $Y$ - i.e., of the matrix algebra $\mathcal{M}$, and of its commutant $\mathcal{M}^{\prime}$, the two mutually commuting sets of imaginary quaternionic units $\ell_{i}$ and $r_{j}$ corresponding to the splitting of the Lie algebra $s o(4)$ of real skew- symmetric $4 \times 4$ matrices into a direct sum of "a left and a right" so(3) Lie subalgebras:

$$
\begin{aligned}
& \ell_{1}=\sigma_{3} \otimes \epsilon, \quad \ell_{2}=\epsilon \otimes \mathbf{1}, \quad \ell_{3}=\ell_{1} \ell_{2}=\sigma_{1} \otimes \epsilon, \\
& \left(\ell_{j}\right)_{\alpha \beta}=\delta_{\alpha 0} \delta_{j \beta}-\delta_{\alpha j} \delta_{0 \beta}-\varepsilon_{0 j \alpha \beta}, \\
& \alpha, \beta=0,1,2,3, j=1,2,3 ; \\
& r_{1}=\epsilon \otimes \sigma_{3}, \quad r_{2}=\mathbf{1} \otimes \epsilon, \quad r_{3}=r_{1} r_{2}=\epsilon \otimes \sigma_{1}
\end{aligned}
$$

where $\sigma_{k}$ are the Pauli matrices, $\epsilon=i \sigma_{2}, \varepsilon_{\mu \nu \alpha \beta}$ is the totally antisymmetric Levi-Civita tensor normalized by $\varepsilon_{0123}=1$. We have

$$
\begin{aligned}
Y\left(x_{1}, x_{2}\right)= & V_{0}\left(x_{1}, x_{2}\right) \mathbf{1}+V_{1}\left(x_{1}, x_{2}\right) \ell_{1}+ \\
& V_{2}\left(x_{1}, x_{2}\right) \ell_{2}+V_{3}\left(x_{1}, x_{2}\right) \ell_{3}= \\
& Y\left(x_{2}, x_{1}\right)^{+} \\
& \left(\ell_{i}^{+}=-\ell_{i},\left[\ell_{i}, r_{j}\right]=0\right) ; \\
V_{\kappa}\left(x_{1}, x_{2}\right)= & \sum_{\substack{m=1 \\
\ell_{0}=1}}^{N}: \varphi_{m}^{\alpha}\left(x_{1}\right)\left(\ell_{\kappa}\right)_{\alpha \beta} \varphi_{m}^{\beta}\left(x_{2}\right) ;,
\end{aligned}
$$

In order to determine the Lie algebra corresponding to the $\mathrm{CR}$ (4.1) in each of the three cases (4.5) we choose a discrete basis and specify the topology of the resulting infinite matrix algebra in such a way that the generators of the conformal Lie algebra (most importantly, the conformal Hamiltonian $H$ ) belong to it. The basis, say $\left(X_{m n}\right)$ where $m, n$ are multiindices, corresponds to the expansion [42] of a free massless scalar field $\varphi$ in creation and annihilation operators of fixed energy states

$\varphi(z)=\sum_{\ell=0}^{\infty} \sum_{\mu=1}^{(\ell+1)^{2}}\left(\left(z^{2}\right)^{-\ell-1} \varphi_{\ell+1, \mu}+\varphi_{-\ell-1, \mu}\right) h_{\ell \mu}(z)$,

where $\left(h_{\ell \mu}(z), \mu=1, \ldots,(\ell+1)^{2}\right)$ form a basis of homogeneous harmonic polynomials of degree $\ell$ in the complex 4-vector $z$ (of the parametrization (2.3) of $\bar{M})$. The generators of the conformal Lie algebra $s u(2,2)$ are expressed as infinite sums in $X_{m n}$ with a finite number of diagonals (cf. Appendix B to [2]). The requirement $s u(2,2) \subset \mathcal{L}$ thus restricts the topology of $\mathcal{L}$ implying that the last (c-number) term in (4.1) gives rise to a non-trivial central extension of $\mathcal{L}$.

The analysis of [2], [3] yields the following

Proposition 4.2 The Lie algebras $\mathcal{L}(\mathbb{F}), \mathbb{F}=\mathbb{R}, \mathbb{C}, \mathbb{H}$ are 1-parameter central extensions of appropriate completions of the following inductive limits of matrix algebras:

$$
\begin{aligned}
& \mathbb{R}: s p(\infty, \mathbb{R})=\lim _{n \rightarrow \infty} s p(2 n, \mathbb{R}) \\
& \mathbb{C}: u(\infty, \infty)=\lim _{n \rightarrow \infty} u(n, n) \\
& \mathbb{H}: s o^{*}(4 \infty)=\lim _{n \rightarrow \infty} s o^{*}(4 n) .
\end{aligned}
$$

In the free field realization (4.4) the suitably normalized central charge coincides with the positive integer $N$.

\footnotetext{
${ }^{3}$ Finite dimensional simple Lie groups $G$ with this property have been extensively studied by mathematicians (for a review and references - see [9]); for an extension to the infinite dimensional case - see [40]. If $Z$ is the centre of $G$ and $K$ is a closed maximal subgroup of $G$ such that $K / Z$ is compact then $G$ is characterized by the property that $(G, K)$ is a hermitean symmetric pair. Such groups give rise to simple space-time symmetries in the sense of [30] (see also earlier work - in particular by Günaydin - cited there).
} 


\section{Fock space representation of the dual pair $\mathcal{L}(\mathbb{F}) \times U(N, \mathbb{F})$}

To summarize the discussion of the last section: there are three infinite dimensional irreducible Lie algebras, $\mathcal{L}(\mathbb{F})$ that are generated in a theory of GCI scalar fields of dimension $d=2$ and correspond to the three real division rings $\mathbb{F}$ (Proposition 4.2). For an integer central charge $N$ they admit a free field realization of type (4.3) and a Fock space representation with (compact) gauge group $U(N, \mathbb{F})$ :

$$
\begin{aligned}
U(N, \mathbb{R}) & =O(N), U(N, \mathbb{C})=U(N), \\
U(N, \mathbb{H}) & =S p(2 N)(=U S p(2 N)) .
\end{aligned}
$$

It is remarkable that this result holds in general.

Theorem 5.1 (i) In any unitary irreducible positive energy representation (UIPER) of $\mathcal{L}(\mathbb{F})$ the central charge $N$ is a positive integer.

(ii) All UIPERs of $\mathcal{L}(\mathbb{F})$ are realized (with multiplicities) in the Fock space $\mathcal{F}$ of $N$ dim $_{\mathbb{R}} \mathbb{F}$ free hermitean massless scalar fields.

(iii) The ground states of equivalent UIPERs in $\mathcal{F}$ form irreducible representations of the gauge group $U(N, \mathbb{F})$ (5.1). This establishes a one-to-one correspondence between UIPERs of $\mathcal{L}(\mathbb{F})$ occurring in the Fock space and the irreducible representations of $U(N, \mathbb{F})$.

The proof of this theorem for $\mathbb{F}=\mathbb{R}, \mathbb{C}$ is given in [2] (the proof of (i) is already contained in [33]); the proof for $\mathbb{F}=\mathbb{H}$ is given in [3].

Remark 5.1 Theorem 5.1 is also valid - and its proof becomes technically simpler - for a 2-dimensional chiral theory (in which the local fields are functions of a single complex variable). For $\mathbb{F}=\mathbb{C}$ the representation theory of the resulting infinite dimensional Lie algebra $u(\infty, \infty)$ is then essentially equivalent to that of the vertex algebra $W_{1+\infty}$ studied in [22] (see the introduction to [2] for a more precise comparison).

Theorem 5.1 provides a link between two parallel developments, one in the study of highest weight modules of reductive Lie groups (and of related dual pairs - see Sect. 1.1) [24, 18, 9, 40] (and [16, 17]), the other in the work of Haag-Doplicher-Roberts [14, 8] on the theory of (global) gauge groups and superselection sectors - see Sect. 1.2. (They both originate - in the talks of Irving Segal and Rudolf Haag, respectively - at the same Lille 1957 conference on mathematical problems in quantum field theory). Albeit the settings are not equivalent the results match. The observable algebra (in our case, the commutator algebra generated by the set of bilocal fields $V_{M}$ ) determines the (compact) gauge group and the structure of the superselection sectors of the theory. (For a more careful comparison between the two approaches - see Sections 1 and 4 of $[2]$.

The infinite dimensional Lie algebra $\mathcal{L}(\mathbb{F})$ and the compact gauge group $U(N, \mathbb{F})$ appear as a rather special (limit-) case of a dual pair in the sense of Howe [16], [17]. It would be interesting to explore whether other (inequivalent) pairs would appear in the study of commutator algebras of (spin)tensor bifields (discussed in Remark 3.3) and of their supersymmetric extension (e.g. a limit as $m, n \rightarrow \infty$ of the series of Lie superalgebras $\operatorname{osp}\left(4 m^{*} \mid 2 n\right)$ studied in [13]).

\section{Acknowledgement}

It is a pleasure to thank my coauthors Bojko Bakalov, Nikolay M. Nikolov and Karl-Henning Rehren: all results (reported in Sects. 3-5) of this paper have been obtained in collaboration with them. I thank Cestmir Burdik for inviting me to talk at the meeting "Selected Topics in Mathematical and Particle Physics", Prague, 5-7 May 2009, dedicated to the 70th birthday of Jiri Niederle. I also acknowledge a partial support from the Bulgarian National Council for Scientific Research under contracts Ph-1406 and DO-02-257.

\section{References}

[1] Bakalov, B., Nikolov, N. M.: Jacobi identities for vertex algebras in higher dimensions, $J$. Math. Phys. 47 (2006) 053505 (30 pp.); math$\mathrm{ph} / 0601012$.

[2] Bakalov, B., Nikolov, N. M., Rehren, K.-H., Todorov, I.: Unitary positive energy representations of scalar bilocal quantum fields, Commun. Math. Phys. 271 (2007) 223-246; math$\mathrm{ph} / 0604069$.

[3] Bakalov, B., Nikolov, N. M., Rehren, K.-H., Todorov, I.: Infinite dimensional Lie algebras of $4 \mathrm{D}$ conformal quantum field theory, J. Phys. A Math. Theor. 41 (2008) 194002; arXiv:0701.0627 [hep-th].

[4] Baumann, K.: There are no scalar Lie fields in three or more dimensional space-time, Commun. Math. Phys. 47 (1976) 69-74.

[5] Borcherds, R.: Vertex algebras, Kac-Moody algebras and the monster, Proc. Natl. Acad. Sci. USA 83 (1986) 3068-3071.

[6] Dobrev, V. K., Mack, G., Petkova, V. B., Petrova, S. G., Todorov, I. T.: Harmonic Analysis on the $n$-Dimensional Lorentz Group and Its Application to Conformal Quantum Field Theory, Lecture Notes in Physics 63, Springer, Berlin 1977. 
[7] Dolan, F. A., Osborn, H.: Conformal four point functions and operator product expansion, Nucl. Phys. B599 (2001) 459-496.

[8] Doplicher, S., Roberts, J.: Why there is a field algebra with a compact gauge group describing the superselection structure in particle physics, Commun. Math. Phys. 131 (1991) 51-107.

[9] Enright, T., Howe, R., Wallach, N.: A classification of uniatry highest weight modules, In Representation Theory of Reductive Groups, Progress in Mathematics 40, Birkhäuser, Basel 1983, pp. $97-143$.

[10] Frenkel, E., Ben-Zvi, D.: Vertex Alegbras and Algebraic Curves, Mathematical Surveys and Monographs 88, AMS 2001; Second ed. 2004.

[11] Frenkel, I. B., Kac, V. G.: Basic representations of affine Lie algebras and dual resonance models, Inventiones Math. 62 (1980) 23-66.

[12] Günaydin, M., Pavlyk, O.: A unified approach to the minimal unitary realizations of noncompact groups and supergroups, JHEP 0609 (2006) 050; hep-th/0604077v2.

[13] Günaydin, M., Scalise, R.: Unitary lowest weight representations of the non-compact supergroup OSp $\left(2 m^{*} \mid 2 n\right)$, J. Math. Phys. 32 (1991) 599-606.

[14] Haag, R.: Local Quantum Physics: Fields, Particles, Algebras, Springer, Berlin 1992, 412 p.

[15] Howe, R.: On the role of the Heisenberg group in harmonic analysis, Bull. Amer. Math. Soc. 3:2 (1980) 821-843.

[16] Howe, R.: Dual pairs in physics: harmonic oscillators, photons, electrons, and singletons, In Applications of Group Theory in Physics and Matehmatical Physics, M. Flato, P. Sally, G. Zuckerman (Eds.) Lectures in Applied Mathematics 21 Amer. Math. Soc., Providence, R.I. 1985, pp. 179-206.

[17] Howe, R.: Remarks on classical invariant theory, Trans. Amer. Math. Soc. 313 (1989) 539-570; Transcending classical invariant theory, J. Amer. Math. Soc. 2:3 (1989) 535-552.

[18] Jakobsen, H. P.: The last possible place of unitarity for certain highest weight module, Math. Ann. 256 (1981) 439-447.

[19] Joseph, A.: Minimal realizations and spectrum generating algebras, Commun. Math. Phys. 36 (1974) 325-338; The minimal orbit in a simple Lie algebra and its associated maximal ideal, Ann. Sci. Ecole Normale Sup. Série 4, 9 (1976) 1-29.
[20] Kac, V. G.: Infinite Dimensional Lie Algebras, Cambridge Univ. Press., Cambridge 1990.

[21] Kac, V.: Vertex Algebras for Beginners, 2nd ed., AMS, Providence, R.I. 1998.

[22] Kac, V., Radul, A.: Representation theory of the vertex algebra $W_{1+\infty}$, Transform. Groups 1 (1996) 41-70.

[23] Kac, V. G., Raina, A. K.: Highest Weight Representations of Infinite Dimensional Lie Algebras, Adv. Series in Math. Phys. 2, World Scientific, Singapore 1987.

[24] Kashiwara, M., Vergne, M.: On the Segal-ShaleWeil representations and harmonic polynomials, Invent. Math 44 (1978) 1-47.

[25] Kazhdan, D., Pioline, B., Wadron, A.: Minimal representations, spherical vectors and exceptional theta series, Commun. Math. Phys. 226 (2002) 1-40; hep-th/0107222.

[26] Kobayashi, T., Mano, G.: The Schrödinger Model, for the Minimal Representation of the Indefinite Orthogonal Group $O(p, q)$, arXiv:0712.1769v2 [math.RT], July 2008 $(167+$ iv pp. $)$.

[27] Lang, S.: Algebra, Third revised editon, Graduate Texts in Mathmatics 211, Sprigner, N.Y. 2002.

[28] Lowenstein, J. H.: The existence of scalar Lie fields, Commun. Math. Phys. 6 (1967) 49-60.

[29] Mack, G.: All unitary representations of the conformal group $S U(2,2)$ with positive energy, Commun. Math. Phys. 55 (1977) 1-28.

[30] Mack, G., de Riese, M.: Simple symmetries: Generalizing conformal field theory, J. Math. Phys. 48 (2007) 052304-1-21; hep-th/0410277.

[31] Mack, G., Todorov, I.: Irreducibility of the ladder representations of $\mathrm{U}(2,2)$ when restricted to the Poincaré subgroup, J. Math. Phys. 10 (1969) 2078-2085.

[32] Nikolov, N. M., Todorov, I. T.: Rationality of conformally invariant local correlation functions on compactified Minkowski space, Commun. Math. Phys. 218 (2001) 417-436; hep-th/0009004.

[33] Nikolov, N. M., Stanev, Ya. S., Todorov, I. T.: Four dimensional CFT models with rational correlation functions, J. Phys. A 35 (2002) 2 985-3 007; hep-th/0110230.

[34] Nikolov, N. M., Stanev, Ya. S., Todorov, I. T.: Globally conformal invariant gauge field theory with rational correlation functions, Nucl. Phys. B670 (2003) 373-400; hep-th/0305200. 
[35] Nikolov, N. M., Rehren, K.-H., Todorov, I. T.: Partial wave expansion and Wightman positivity in conformal field theory, Nucl. Phys. B722 (2005) 266-296; hep-th/0504146.

[36] Nikolov, N. M., Rehren, K.-H., Todorov, I.: Harmonic bilocal fields generated by globally conformal invariant scalar fields, Commun. Math. Phys. 279 (2008) 225-250; arXiv:0711.0628 [hep-th].

[37] Nikolov, N. M.: Vertex algebras in higher dimensions and globally conformal invariant quantum field theory, Commun. Math. Phys. 253 (2005) 283-322; hep-th/0307235.

[38] Nikolov, N. M., Todorov, I. T.: Elliptic thermal correlation functions and modular forms in a globally conformal invariant QFT, Rev. Math. Phys. 17 (2005) 613-667; hep-th/0403191.

[39] Robinson, D. W.: On a soluble model of relativistic field theory, Physics Lett. 9 (1964) 189-190.

[40] Schmidt, M. U.: Lowest weight representations of some infinite dimensional groups on Fock spaces, Acta Appl. Math. 18 (1990) 59-84.

[41] Streater, R. F., Wightman, A. S.: PCT, Spin and Statistics, and All That, W. A. Benjamin, Reading 1964; Princeton University Press, Princeton 2000 .
[42] Todorov, I. T.: Infinite-dimensional Lie algebras in conformal QFT models, in: A.O. Barut, H.D. Doebner (eds.), Conformal Groups and Related Symmetries. Physical Results and Mathematical Background, pp. 387-443, Lecture Notes in Physics 261, Springer, Berlin 1986.

[43] Uhlmann, A.: The closure of Minkowski space, Acta Phys. Pol. 24 (1963) 295-296.

[44] Weil, A.: Sur certains groupes d'opérateurs unitaires, Acta Math. 111 (1964) 143-211; Sur la formule de Siegel dans la théorie des groupes classiques, ibid bf 113 (1965) 1-87.

[45] Wick, G. C., Wightman, A. S., Wigner, E. P.: The intrinsic parity of elementary particles, Phys. Rev. 88 (1952) 101-105.

[46] Zhu, Y.: Modular invariance of characters of vertex operator algebras, J. Amer. Math. Soc. 9:1 (1996) 237-302.

Ivan Todorov

E-mail: todorov@inrne.bas.bg

Institute for Nuclear Research and Nuclear Energy,

Tsarigradsko Chaussee 72

BG-1784 Sofia, Bulgaria 